\title{
C-ARM DISTORTION CORRECTION USING PATIENT CT AS A FIDUCIAL
}

\author{
Gouthami Chintalapani, Russell H. Taylor \\ Department of Computer Science \\ Johns Hopkins University
}

\begin{abstract}
$\mathrm{X}$-ray imaging is an extensively used intra-operative imaging modality because of its low cost and portability. These images have to be corrected for geometric distortion in order to use them for quantitative analysis. Current distortion correction techniques, which use a grid pattern phantom, are not user friendly, for example the phantom interferes with the patient anatomy being imaged. We present a novel method to estimate the c-arm distortion parameters along with the patient pose using patient $\mathrm{CT}$ as a fiducial. This method could be very useful in applications such as $3 \mathrm{D}$ reconstruction and surgical navigation. In this method, we characterize the c-arm distortion patterns statistically using principal component analysis. The distortion correction method optimizes the distortion parameters by comparing the fluoroscopic images and the projections of the registered patient CT. Our simulation experiments show that the distortion parameters can be recovered up to an average accuracy of $0.5117 \mathrm{~mm}$ with a pose error of about $0.1692 \mathrm{~mm}$ in translation, $0.2112 \mathrm{deg}$ in rotation.
\end{abstract}

\section{INTRODUCTION}

Fluoroscopic images are extensively used for intra-operative imaging analysis because of the relatively low cost and ease of operation of the x-ray fluoroscope. These images suffer from pose dependent geometric distortion mainly due to the curved nature of detector and earth's magnetic field [1]. In order to perform quantitative analysis in applications such as surgical navigation or $3 \mathrm{D}$ reconstruction, these images need to be corrected for distortion.

Current distortion correction techniques, for example, use a calibration phantom with metal BBs $[1,2]$ or grooves implanted [3] in certain geometry. All these methods characterize the distortion by fitting a high-order polynomial between the observed point or line features of the grid in the x-ray image to the physical geometrical coordinates of the respective phantom grid. These phantoms are easy to use offline, but might be cumbersome when used for intra-operative distortion correction. The disadvantages being: 1)the grid interferes with the imaged patient anatomy if left on the image intensifier while imaging; 2) the c-arm has to be tracked for pose dependent distortion correction; and 3) increased dose and time if two image sets are taken, one with and the other without the phantom. [1] proposes to interpolate the distortion parameters from the neighboring poses, to avoid the phantom interference with the patient anatomy. But this requires the c-arm to be tracked accurately to determine the pose. Yao et al. [3] propose to use the same c-arm poses intra-operatively for which the distortion was measured offline. This procedure works for few images, could be time-consuming when collecting a lot of data and the c-arm must be repeatable.

Our approach to address these issues is to use patient CT images as a fiducial for both distortion correction and c-arm tracking. [4] presents preliminary work on c-arm pose recovery using patient specific model as fiducial. In this paper, we propose a novel method to perform simultaneous distortion correction and pose estimation using patient $\mathrm{CT}$. This method uses prior knowledge in two forms 1) patient CT and 2)statistical characterization of the distortion patterns. C-arm distortion variations are characterized with principal component analysis. We then register the patient $\mathrm{CT}$ to a set of X-ray images, create projections of the registered $\mathrm{CT}$, and then recover the distortion parameters by comparing the actual X-ray images to the CT projections as shown in Fig 1.

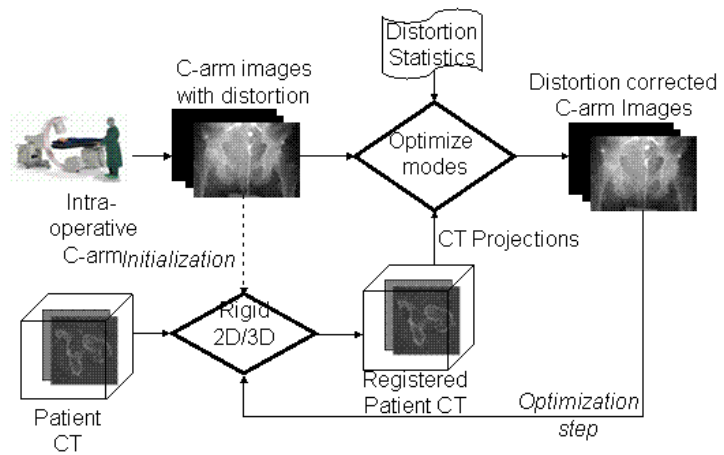

Fig. 1. Flowchart showing simultaneous pose estimation and distortion correction

There has been extensive prior work on rigid 2D/3D registration between the patient CT and the x-ray images [5]. Currently, we use a mutual information based rigid $2 \mathrm{D} / 3 \mathrm{D}$ registration method developed by Sadowsky et al. [6], an extension of Yao et al. [7], although any rigid 2D/3D registration 
method would satisfy our requirements in this work. The rest of the paper is organized as follows. Section 2 discusses the principal component analysis of the distortion patterns and the algorithmic aspects of the distortion correction method. Simulation results are given in section 3 and conclusions and future work in section 4 .

\section{METHOD}

\subsection{Statistical Characterization of $\mathbf{C}$-arm Distortion}

Fluoroscopic images of the phantom grid are used to measure c-arm distortion patterns. $\mathrm{C}$-arm distortion is modelled with a high-order polynomial that maps the observed phantom grid coordinates from the image extracted through segmentation $\left(u_{d}, v_{d}\right)$ to the nominal coordinates from the physical geometry of the phantom $\left(u_{o}, v_{o}\right)$. The distortion vector, defined for each grid point, is given by

$$
\triangle \vec{d}=(\triangle u, \triangle v)=\left(u_{d}, v_{d}\right)-\left(u_{0}, v_{0}\right)
$$

The array of such vectors for all the grid points is the distortion map of that image. These grid coordinates define the control points to parameterize the distortion using a fifth-order Bernstein polynomial $(n=5)$ as shown in the following equation.

$$
\left(u_{d}, v_{d}\right)=\sum_{i=0}^{n} \sum_{j=0}^{n} C_{i j} B_{i j}\left(u_{0}, v_{0}\right)
$$

where

$$
B_{i j}\left(u_{0}, v_{0}\right)=\left(\begin{array}{c}
n \\
i
\end{array}\right) u_{0}^{i}\left(1-u_{0}\right)^{n-i}\left(\begin{array}{c}
n \\
j
\end{array}\right) v_{0}^{j}\left(1-v_{0}\right)^{n-j}
$$

This can be written in the matrix form as follows.

$$
\left(U_{o b s}\right)_{m \times 2}=\left(B\left(U_{n o m}\right)\right)_{m \times(n+1)^{2}} \times C_{(n+1)^{2} \times 2}
$$

where $U_{o b s}$ is the array of observed grid coordinates in the image; $U_{n o m}$ is the array of physical grid coordinates; and $m$ being number of grid points on the phantom (here $m=300$ ).

The coefficients of the polynomial $C$ matrix or $C_{i j}$ are computed in the least squares sense. This polynomial is then used to interpolate the distortion at all image pixels to create a dewarped image. For statistical analysis, data matrix is created by stacking the distortion maps of all the images in one matrix as given below.

$$
\Delta=\left(\begin{array}{cccccc}
\triangle u_{11} & \triangle u_{12} & \cdot & \cdot & \cdot & \triangle u_{1 k} \\
\cdot & \cdot & \cdot & \cdot & \cdot & \cdot \\
\cdot & \cdot & \cdot & \cdot & \cdot & \cdot \\
\triangle u_{m 1} & \triangle u_{m 2} & \cdot & \cdot & \cdot & \triangle u_{m k} \\
\triangle v_{11} & \triangle v_{12} & \cdot & \cdot & \cdot & \triangle v_{1 k} \\
\cdot & \cdot & \cdot & \cdot & \cdot & \cdot \\
\cdot & \cdot & \cdot & \cdot & \cdot & \cdot \\
\triangle v_{m 1} & \triangle v_{m 2} & \cdot & \cdot & \cdot & \Delta v_{m k}
\end{array}\right)
$$

where $\left(\triangle u_{i j}, \triangle v_{i j}\right)$ is the distortion vector of the $i_{t h}$ grid point in the $j_{t h}$ image; $m$ is the number of grid points on the phantom; and $k$ is the number of images (in our case, $k=100)$.

The mean distortion map $M_{0}$ is given by the average of the columns of the $\Delta$ matrix. Principal component analysis is performed on the zero mean (subtract mean distortion map) data matrix to extract the principal modes of variation. This analysis enables us to express any distortion map as a linear combination of the principal modes.

$$
\triangle \vec{d}=M_{0}+\sum_{i=1}^{n} \lambda_{i} D_{i}
$$

where $M_{0}$ is the mean distortion map and $D_{i}$ is the $i^{t h}$ principal component. The intial characterization of distortion with Bernstein polynomials has 72 parameters for a fifth-order polynomial, where as the principal component analysis shows that 3-5 parameters are sufficient to characterize the same distortion, thereby significantly reducing the dimensionality of the problem. These results indicates that the $\mathrm{C}$-arm distortion patterns although appear to be random, can be predicted fairly accurately with this model. This parameterization of distortion function does not need any pose information for correction which in turn avoids $\mathrm{C}$-arm tracking which is timeconsuming and expensive.

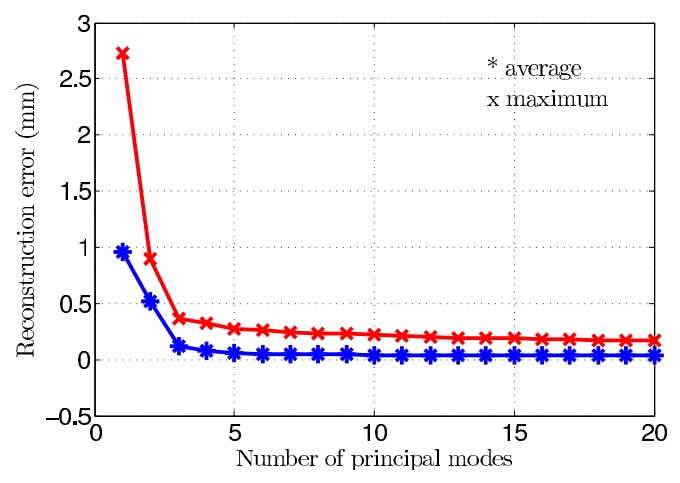

Fig. 2. Number of principal components required to recover distortion parameters. Our experiments have shown that the first three modes capture about $99 \%$ of the variation [8].

\subsection{Pose Estimation and Distortion Correction}

The following steps explain the two step optimization setup for recovering the distortion parameters. The image is given as a tuple $I_{i}^{j}, F_{i}, \triangle d_{i}$ where $I_{i}^{j}$ are fluoroscopic images in $j^{t h}$ view, $F_{i}$ is the patient pose and $\triangle d_{i}$ is the estimated distortion map in $i^{t h}$ iteration. We assume that the c-arm is calibrated for intrinsic projective parameters and the trajectory of the carm is known. $I_{0}^{j}, F_{0}, M_{0}$ is the input to the optimizer where 
$I_{0}^{j}$ are the actual distorted x-ray images, $F_{0}$ is the intial estimate of the pose and $M_{0}$ is the mean distortion map.

1. Update the patient pose by computing the $2 \mathrm{D} / 3 \mathrm{D}$ rigid transformation between the patient model and the $\mathrm{x}$ ray images. This is achieved by maximizing the mutual information based similarity measure between the $\mathrm{x}$-ray images and the projections of the patient model. In our $2 \mathrm{D} / 3 \mathrm{D}$ registration method, we use a tetrahedral mesh approximation of the patient $\mathrm{CT}$ as our $3 \mathrm{D}$ model [6]. This method uses downhill simplex optimization method.

$$
F_{i+1}=\arg \max _{F} \sum_{j} M I\left(I_{i}^{j}, D R R\left(F_{i}\right)\right)
$$

2. Apply the transformation obtained in the previous step and create the projections (DRRs) $I_{p}^{j}$ of the registered patient specific model.

$$
I_{p}^{j}=\operatorname{Project}\left(F_{i+1} * \text { Model, } \text { Pose }^{j}\right)
$$

3. Estimate the distortion parameters by searching the mode weight parameter space to optimize the similarity measure between the $2 \mathrm{D}$ projections of the $3 \mathrm{D}$ model and the $\mathrm{x}$-ray images.

$$
\lambda_{\text {opt }}^{j}=\arg \min _{\lambda} S S D\left(I_{p}^{j}, \operatorname{Dewarp}\left(I_{0}^{j}, M_{0}, \lambda^{j}\right)\right)
$$

For dewarping any image, compute the estimated observed grid coordinates for each image as follows and the polynomial coefficients as given in 2 .

$$
U_{\text {obs }}=U_{\text {nom }}+M_{0}+D \times \lambda
$$

4. Dewarp the distorted $x$-ray images using the optimal mode parameters computed in the previous step.

$$
I_{i+1}^{j}=\operatorname{DewarpImage}\left(I_{0}^{j}, M_{0}, \lambda_{\text {opt }}^{j}\right)
$$

Note that the actual x-ray $\left(I_{0}^{j}\right)$ images are used in dewarping where as the dewarped images $\left(I_{i}^{j}\right)$ in each iteration are used to estimate the pose. Iterate steps 1 through 4 until convergence with $i=i+1$.

\section{EXPERIMENTS AND RESULTS}

This section discusses the experimental setup and the results of our simulation experiments. For statistical characterization of the c-arm distortion, we have taken 120 images of the grid phantom, one, approximately, every 3 degrees, along the propeller axis of an OEC $9600 \mathrm{c}$-arm. We corrected all these images using the grid features with polynomial interpolation and also computed the distortion map for all these images. Out of the 120 images, we left out 20 images from the PCA analysis to perform leave-out validation experiments. For the left out images, we also computed the inverse warp functions. PCA analysis on the distortion maps have shown that the first three principal components capture about $99 \%$ of the variation [8]. We have generated 6 DRRs in $[0,30,60,90,135,150]$ views for 2D/3D registration. Note that the DRRs are generated from the actual CT volume where as the model we use in $2 \mathrm{D} / 3 \mathrm{D}$ registration is a tetrahedral mesh approximation of the CT. These DRRs were warped using the inverse warp functions to simulate the actual $\mathrm{x}$-ray distortion. We have used both $\mathrm{C}++(2 \mathrm{D} / 3 \mathrm{D}$ registration) and MATLAB (dewarping) for our experiments.
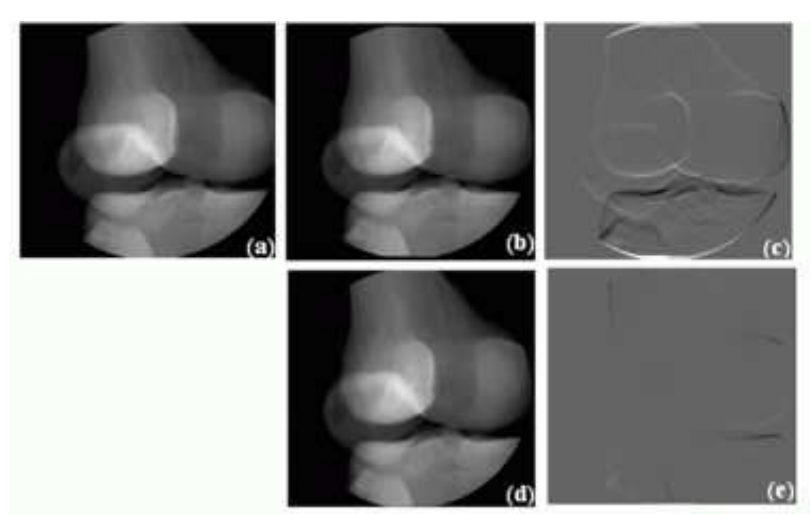

Fig. 3. Results from simulation experiments. (a) true projection; (b) warped projection (simulated x-ray); (c) difference between true and warped projection ((a) - (b)); (d) registered and distortion corrected projection; (f) (a) - (d); The bottom row shows the distortion map before and after correction.

The algorithm converges in 3 to 4 iterations. The registration results are shown in Fig 3. The figure shows the difference in the estimated dewarped image and the actual x-ray image and also the residual distortion map. The bright regions in (c) is due to the interpolation error in creating inverse warp functions. The quantitative analysis is shown in Table 1. The average distortion before correction is about $2.12 \mathrm{~mm}$ and a maximum distortion of $6.4 \mathrm{~mm}$ with a pixel size of $0.44 \mathrm{~mm}$ per pixel. The residual error in distortion map is the $L 2$ norm of the actual distortion map estimated from the phantom grid and the estimated distortion map from our method.

\section{DISCUSSION}

We have shown that we can recover the distortion parameters up to an average accuracy of $0.5112 \mathrm{~mm}$ and simultaneously 


\begin{tabular}{|l|l|l|l|l|l|l|l|l|c|}
\hline & \multicolumn{4}{|c|}{$\begin{array}{c}\text { Pose Estimation Error } \\
(\mathrm{mm}, \mathrm{deg})\end{array}$} & \multicolumn{3}{c|}{$\begin{array}{c}\text { Distortion Error } \\
(\mathrm{mm})\end{array}$} \\
\cline { 2 - 11 } & $T_{a v g}$ & $T_{\text {std }}$ & $T_{\max }$ & $R_{\text {avg }}$ & $R_{\text {std }}$ & $R_{\max }$ & avg & std & max \\
\hline $\begin{array}{l}\text { Unknown Pose, } \\
\text { Known Distortion }\end{array}$ & 0.0050 & 0.0065 & 0.0217 & 0.0069 & 0.0113 & 0.0397 & N/A & N/A & N/A \\
\hline $\begin{array}{l}\text { Known Pose, Un- } \\
\text { known Distortion }\end{array}$ & N/A & N/A & N/A & N/A & N/A & N/A & 0.3309 & 0.1742 & 0.7861 \\
\hline $\begin{array}{l}\text { Unknown Pose, Un- } \\
\text { known Distortion }\end{array}$ & 0.1692 & 0.1996 & 0.6546 & 0.2112 & 0.1875 & 0.4682 & 0.5117 & 0.2915 & 3.2823 \\
\hline
\end{tabular}

Table 1. Pose error and residual error in distortion correction from simulation experiments. Columns 2-4 represent the mean, standard deviation and the maximum error in estimating the translation and columns 5-7 for rotation respectively. The first row shows the pose error when the distortion is known from 6 simulations. The second row shows the residual distortion correction error from 20 experiments and the third row shows the pose and distortion error in simultaneous pose estimation and distortion correction from 6 simulation experiments.

estimate the pose with an accuracy of $0.1692 \mathrm{~mm}$ in translation and 0.2112 degrees in rotation. These results are promising as our distortion correction method relies on the patient CT information as compared to the external phantom based methods. The maximum error of $3.2823 \mathrm{~mm}$ is the maximum of maximum distortion error in all the images. These errors can be attributed to the amount of bone present in the $\mathrm{x}$-ray images. Since the distortion map is defined all over the image with the grid points, there isn't enough information to constrain all the points. This method is not yet feasible for intra-operative use as the $2 \mathrm{D} / 3 \mathrm{D}$ registration step is time consuming (approx 20 minutes). We are currently exploring other faster $2 \mathrm{D} / 3 \mathrm{D}$ registraton methods that would decrease the run-time. Real data experiments are currently underway. We would like to extend this method to do c-arm tracking using patient information as well. A Further extension would be to use statistical bone atlas instead of patient CT. The intuition here is that if the anatomical shape variations of the patient bone and the c-arm distortion patterns lie in different subspaces, we can recover distortion parameters using a statistical bone atlas. However, these preliminary results support our hypothesis to recover the distortion parameters with the patient $\mathrm{CT}$ as a fiducial and hence can serve as a starting point for future research.

\section{ACKNOWLEDGMENTS}

We would like to thank Ameet Jain, Ofri Sadowsky, and Dr. Jerry Prince for their assistance in this work. This work was supported in part by NSF ERC Grant EEC9731478 and by NIH/NIBIB Research Grant R21-EB003616.

\section{REFERENCES}

[1] R. Fahrig, M. Moreau, and D.W. Holdsworth, "Threedimensional computed tomographic reconstruction using a c-arm mounted xrii: correction of image intensifier distortion," Med Phys, vol. 24(7), pp. 1097-106, 1997.

[2] H. Livyatan, Z. Yaniv, and L. Joskowicz, "Robust automatic c-arm calibration for fluoroscopy-based navigation: A practical approach," in MICCAI, 2002, vol. 2489, pp. $60-68$.

[3] J. Yao, R.H. Taylor, R.P. Goldberg, R. Kumar, A. Bzostek, R.V. Vorhis, P. Kazanzides, and A. Gueziec, "Robust automatic c-arm calibration for fluoroscopybased navigation: A practical approach," in Computer Aided Surgery (CAS), 2000, vol. 5(6), pp. 373-90.

[4] O. Sadowsky, K. Ramamurthi, J.L. Prince, and R.H. Taylor, "Using anatomical models and fast rendering algorithms for c-arm pose recovery and cone-beam tomographic reconstruction of bone anatomy," in International Society for Computer Assisted Orthopaedic Surgery (CAOS), 2005.

[5] B.A. Maintz and M.A. Viergever, "A survey of medical image registration," Medical Image Analysis (MedIA), vol. 2(1), pp. 1-36, 1998.

[6] O. Sadowsky, J.D. Cohen, and R.H. Taylor, "Rendering tetrahedral meshes with higher-order attenuation functions for digital radiograph reconstruction," in IEEE Visualization 2005, 2005.

[7] J. Yao, "A statistical bone density atlas and deformable medical image registration," Ph. D. Thesis, The Johns Hopkins Univeristy, 2002.

[8] G. Chintalapani, A.K. Jain, and R.H. Taylor, "Statistical characterization of c-arm distortion with application to intra-operative distortion correction," To appear in SPIE Medical Imaging; Visualization, Image-Guided Procedures, and Display, 2007. 